\title{
Clinical Change by Non-Invasive Imaging Procedure
}

National Cancer Institute

\section{Source}

National Cancer Institute. Clinical Change by Non-Invasive Imaging Procedure. NCI

Thesaurus. Code C123443.

Any clinical change to a subject as determined by a non-invasive imaging procedure. 